Reprod. Nutr. Dévelop., 1988, 28 (1), 149-150.

\title{
Influence de la nature de la source azotée (tourteau d'arachide ou urée) sur l'activité microbienne mesurée in vitro
}

Marie-Claude HILLAIRE, J. P. JOUANY, S. BA $\left({ }^{*}\right), Y$, GEAY $(*)$

Laboratoire de la Digestion des Ruminants

(*) Laboratoire de la Production de Viande

I.N.R.A., Theix, 63122 Ceyrat, France.

Summary. Activity of rumen microbes was estimated from the production of fermentation end products in a batch system inoculated with rumen contents from two groups of animals fed the same amount of PDIMN from two different sources of nitrogen (groundnut meal or urea). Our results suggest that nitrogen from groundnut meal is used more efficiently by rumen microbes than urea, which agrees with growth trials conducted simultaneously on bulls.

Certains travaux ont montré que la synthèse microbienne était plus importante à partir d'acides aminés provenant de protéines dégradables que de l'ammoniaque issue d'azote uréique (Blake et al., 1983). Une expérimentation a été réalisée pour étudier l'influence de la nature de la source azotée (tourteau d'arachide ou urée) sur les performances de taurillons lorsque les apports théoriques d'azote fermentescible sont identiques. Nous présentons ci-dessous les résultats des mesures in vitro de l'activité fermentaire des microbes du rumen, adaptés à chacune des deux formes d'azote testées, à partir d'inocula prélevés à l'abattage de ces animaux.

Matériel et méthodes. Deux lots de 12 taurillons Charolais $\times$ Salers ont reçu respectivement, depuis l'âge de 8 mois jusqu'à l'abattage à 17 mois $(550 \mathrm{~kg}$ de poids vif), 2 régimes à base d'ensilage de maïs ( $77 \%$ de matière sèche ingérée) complémentés, à même niveau d'apport d'azote fermentescible (PDIMN), soit par du tourteau d'arachide (A) $(12 \%$ de la MS), soit par de l'urée (U) $(1,3 \%$ de la MS). Ce niveau a été ajusté à l'apport d'énergie (PDIMN = PDIME), identique dans les 2 lots. Du tourteau tanné (50\% de soja ; $50 \%$ de colza) et de l'amidon, ont permis, par ailleurs, d'équilibrer les apports totaux de protéines digestibles dans l'intestin (PDIN = PDIE). Un complément minéral et vitaminique a été distribué à tous les animaux. Il a notamment permis de maintenir la teneur en $S$ de la ration au-dessus de $1,3 \mathrm{~g} / \mathrm{kg} \mathrm{MS}$.

A l'abattage, nous avons prélevé du contenu de rumen sur 5 animaux de chaque lot qui étaient à jeun depuis 16 à $18 \mathrm{~h}$. Le contenu a été rapidement placé dans le système d'incubation in vitro décrit par Jouany et Thivend (1986) pour constituer l'inoculum A ou l'inoculum U.

On y ajoute une solution tampon dont la composition minérale est voisine de celle de la salive. L'inoculum $A$ reçoit $3,2 \mathrm{~g}$ de $A$ et $12,8 \mathrm{~g}$ d'amidon de blé, alors que $267,9 \mathrm{mg}$ d'U et $14,1 \mathrm{~g}$ d'amidon de blé sont introduits dans l'inculum $U$. Les quantités de matière organique fermentescible et $d^{\prime}$ 'azote fermentescible étaient ainsi identiques pour les deux types d'inoculum. L'activité microbienne a été appréciée à partir des principaux paramètres fermentaires mesurés pendant une durée de $5 \mathrm{~h}$. 
Résultats et discussion. (Tableau 1). La quantité de matière organique fermentée par l'inoculum $A$ en présence du substrat $A$ et calculée selon la méthode de Demeyer et Van Nevel (1975) a été plus élevée $(P<0,05)$ que celle fermentée par l'inoculum $U$ alimenté avec $U$. II en a été de même pour la production des acides gras volatils (AGV) (différence non significative). Ce résultat est corroboré par la plus faible valeur du $\mathrm{pH}(\mathrm{P}<0,05)$ et la plus grande production de gaz $(P<0,01)$ observée dans les fermenteurs $A$ alimentés avec $A$. La proportion des acides butyrique et valérique sous forme linéaire ou ramifiée, a été significativement plus élevée dans ces fermenteurs $A$. La désamination de certains acides aminés de $A$ est probablement à l'origine de l'augmentation $(\mathrm{P}<0,05)$ de la proportion des AGV ramifiés $\left(\mid \mathrm{C}_{4}\right.$ et $\left.\mid \mathrm{C}_{5}\right)$. La méthanogenèse a été significativement plus importante dans les fermenteurs ensemencés avec $A$ et alimentés avec le substrat $A$, alors que la concentration en ammoniac $\left(\mathrm{N}-\mathrm{NH}_{3}\right)$ du liquide au temps $5 \mathrm{~h}$ était plus faible.

Nos résultats suggèrent qu'à apport d'azote fermentescible et d'énergie égal, I'inoculum $A$ recevant le substrat $A$ a une activité fermentaire plus intense que l'inoculum $U$ recevant $U$, bien que l'apport d'amidon ait été plus important dans le dernier cas. II en est probablement de même de la synthèse microbienne. La plus faible concentration en $\mathrm{N}-\mathrm{NH}_{3}$ du liquide des fermenteurs ensemencés avec $\mathrm{A}$ et alimentés avec $A$ résulte probablement d'une fixation d'azote bactérien plus importante puisque les apports d'azote fermentescible, déterminés par une expérimentation préalable à l'aide du même dispositif in vitro, étaient les mêmes pour $A$ et $U$. Ainsi, ces résultats peuvent expliquer la croissance plus élevée des taurillons ayant reçu du tourteau d'arachide par rapport à celle de leurs homologues ayant reçu l'urée $(1423 \pm 178$ contre $1330 \pm 118 \mathrm{~g} / \mathrm{j})$, bien que l'écart observé ne soit pas significatif.

TABL. 1. - Influence de la nature de l'azote sur les paramètres fermentaires mesurés in vitro.

\begin{tabular}{|c|c|c|c|c|c|c|c|c|c|c|c|c|}
\hline \multirow{2}{*}{ Azote } & \multirow{2}{*}{$\begin{array}{c}\mathrm{AGV} \\
(\mathrm{mM} / 1)\end{array}$} & \multirow{2}{*}{$\begin{array}{c}\text { MOF } \\
(\mathrm{g})\end{array}$} & \multirow{2}{*}{$\mathrm{pH}$} & $\mathrm{C}_{2}$ & $\mathrm{C}_{3}$ & $\mathrm{IC}_{4}$ & $\mathrm{C}_{4}$ & $\mathrm{IC}_{5}$ & $\mathrm{C}_{5}$ & \multirow{2}{*}{$\begin{array}{l}\text { gaz } \\
\text { (1) }\end{array}$} & \multirow{2}{*}{$\begin{array}{l}\mathrm{CO}_{2} \\
\mathrm{CH}_{4}\end{array}$} & \multirow{2}{*}{$\begin{array}{l}\mathrm{N}-\mathrm{NH}_{3} \\
(\mathrm{mg} / 1)\end{array}$} \\
\hline & & & & \multicolumn{6}{|c|}{ (p. 100) } & & & \\
\hline $\begin{array}{l}\text { Arachide } \\
\text { Urée } \\
\text { Signification }\end{array}$ & $\begin{array}{l}145,3 \\
129,0\end{array}$ & $\begin{array}{l}5,2 \\
4,5\end{array}$ & $\begin{array}{l}5,1 \\
5,5\end{array}$ & $\begin{array}{l}66,5 \\
68,7\end{array}$ & $\begin{array}{l}14,9 \\
16,8\end{array}$ & $\begin{array}{l}0,59 \\
0,42\end{array}$ & $\begin{array}{l}15,2 \\
11,8\end{array}$ & $\begin{array}{l}0,80 \\
0,53\end{array}$ & $\begin{array}{l}1,2 \\
0,9\end{array}$ & $\begin{array}{l}1,8 \\
1,6\end{array}$ & $\begin{array}{l}3,2 \\
3,7\end{array}$ & $\begin{array}{l}104,5 \\
228,8\end{array}$ \\
\hline statistique & NS & $*$ & * & NS & NS & $*$ & * & $*$ & $* *$ & $* *$ & * & * \\
\hline
\end{tabular}

AGV : Acides gras volatils : MOF : Matière organique fermentée ; $C_{2}$ : acétate ; $C_{3}:$ proprionate $; C_{4}$ : butyrate ; $\mathrm{C}_{5}$ : valérate; $\mathrm{N}-\mathrm{NH}_{3}$ : azote ammoniacal; $\mathrm{NS}$ : différence non significative; ${ }^{*} \mathrm{P}<0,05$; ** $\mathrm{P}<0,01$.

Blake J. S., Salter D. N., Smith R. H., 1983. Br. J. Nutr., 50, 769-782.

Demeyer D. I., Van Nevel C. J., 1975. In Digestion and metabolism in the ruminant. I. W. McDonald and A.C.I. Warner ed. The University of New England Publ. Unit., 366-382.

Jouany J. P., Thivend. P., 1986. Anim. Feed Sci. Technol., 215-229. 